\title{
Less is more: Morphometric and psychological differences between low and high reappraisers
}

\author{
Edoardo Pappaianni ${ }^{1}$ (1) - Nicola De Pisapia ${ }^{1} \cdot$ Roma Siugzdaite $^{2} \cdot$ Cristiano Crescentini $^{3} \cdot$ Antonio Calcagnì $^{1,4}$. \\ Remo Job ${ }^{1}$ - Alessandro Grecucci ${ }^{1}$
}

Published online: 19 December 2019

(C) The Psychonomic Society, Inc. 2019

\begin{abstract}
Emotion regulation plays a crucial role in an individual's well-being, as it is known that deficits in regulating emotions can lead to psychological and psychiatric disorders. Cognitive reappraisal is widely considered to be an adaptive and effective emotionregulation strategy. People are more or less able to apply it, but it is still not clear how reappraisal affects brain structures and the psychological profile of individuals. In our study we thus aimed to explore the impact of applying reappraisal at both the neural and the psychological level. Source-based morphometry (SBM), a whole-brain multivariate method based on the Independent Component Analysis that extracts patterns of covariation of gray matter ("independent networks"), was applied to the MRI images of 37 participants. In order to enrich their psychological profiles, we measured their experienced affectivity (PANAS) and their empathic abilities (IRI). Based on the frequency of applying reappraisal (ERQ), participants were divided into low and high reappraisers (18 vs. 19). An independent source of gray matter emerged as being different between the groups: specifically, low reappraisers showed more gray matter volume concentration in a network including the frontal, temporal, and parietal regions as compared to high reappraisers. At the psychological level, low reappraisers reported a more strongly experienced negative affect, while no difference among reappraisers emerged with regard to empathic abilities. Capitalizing on a multivariate method for structural analysis that is innovative in this field, this study extends previous observations on individual differences in the ability to regulate emotions, and it describes a plausible impact of reappraisal on brain structures and affectivity.
\end{abstract}

Keywords Reappraisal $\cdot$ Morphometry $\cdot$ Multivariate $\cdot$ Affectivity $\cdot$ Emotion regulation

\section{Introduction}

Research has shown that the ability to regulate emotion is a determining factor for individual and interpersonal well-be-

Electronic supplementary material The online version of this article (https://doi.org/10.3758/s13415-019-00757-5) contains supplementary material, which is available to authorized users.

Edoardo Pappaianni

edoardo.pappaianni@unitn.it

1 Department of Psychology and Cognitive Sciences, University of Trento, Trento, Italy

2 MRC Cognition and Brain Sciences Unit, University of Cambridge, Cambridge, UK

3 Department of Languages and Literature, Communication, Education and Society, University of Udine, Udine, Italy

4 Department of Social and Developmental Psychology, University of Padua, Padua, Italy ing, whereas a deficit in this capacity leads to several disturbances (Sheppes et al., 2015). According to Gross (cf. Gross, 1999), emotion regulation refers to a group of strategies through which "individuals influence which emotions they have, when they have them, and how they experience and express these emotions." Largely neglected in the past, emotional regulation has become an increasingly investigated research topic because of its potential clinical implications (Gross, 2017). While we know that individuals regulate their emotions with a variety of strategies, investigators have focused their attention on only a few of them. One of the most studied strategies is cognitive reappraisal (CR). CR is defined as a cognitive interpretation of an emotional stimulus in order to alter its impact (Gross \& John, 2003). CR is considered as an antecedent-focused emotion-regulation strategy, since it works immediately before the emotional experience. Research has showed that CR is adaptive and has beneficial effects, as it is linked to more positive emotions, general higher social functioning, and better individual well-being (Gross \& John, 2003). 
In addition, $\mathrm{CR}$ has been shown to produce larger beneficial effects on memory when compared to other strategies such as, for example, expressive suppression (Dörfel et al., 2014; Hayes et al., 2010; Webb et al., 2012). Expressive suppression is a response-focused emotion-regulation strategy that works on the inhibition of emotion-expressive behavior (Gross, 1998; Gross \& John, 2003). Contrary to CR, expressive suppression has been shown to produce detrimental effects on well-being and memory, and it increases physiological indexes of arousal (Gross, 2002; Gross \& John, 2003). Notably, difficulties in reappraising emotions seem to lead to several psychopathological conditions (Dadomo et al., 2018; De Panfilis et al., 2019; Kring \& Werner, 2004).

\section{Neural bases of cognitive reappraisal}

In recent years, the neural substrates of $C R$ have received quite a lot of attention (Burklund et al., 2014; Cutuli, 2014; Etkin et al., 2015; Grecucci et al.,2013a, b, 2019; Goldin et al., 2008, 2009; Kanske et al., 2011; McRae et al., 2010, 2012). At the functional level, CR increases the activity of the dorsolateral prefrontal cortex (DLPFC) (effect of strategy), and it decreases the activation of emotion-generation structures such as, for example, the amygdala or the insula (effect of regulation) (Buhle et al., 2014; Cutuli, 2014; Grecucci et al., 2013a, b; Ochsner \& Gross, 2005; Ochsner et al., 2012; Banks et al., 2007). Moreover, the activity of DLPFC during a reappraisal task positively correlates with the frequency of usage of CR (Grecucci et al., 2013a) as measured by the Emotion Regulation Questionnaire (ERQ; Gross \& John, 2003), confirming a link between DLPFC and CR. Notably, ERQ negatively correlates with the activity of the amygdala (Drabant et al., 2009) and of the right anterior insula (Carlson \& Mujica-Parodi, 2010). In a more recent study, using a "Cued Emotional Conflict Task," individuals who used CR more frequently displayed a stronger fronto-cingulate activity during the inhibition of conflictual responses related to sad faces, whereas no relation was found between these regions and other strategies (as expressive suppression) (Vanderhasselt et al., 2013). Besides the frontal areas, Hayes et al. (2010) reported other key areas found to be active when reappraising emotional stimuli, such as the left paracingulate gyrus, the middle temporal gyrus, and portions of the parietal cortex. Indeed, in a recent literature review on the neural bases of emotion regulation, the most significant clusters of brain activations were localized in the parietal and temporal semantic areas (Messina et al., 2015; see also Grecucci et al., 2013a,b; Messina et al., 2016; Viviani, 2013).

The neural bases of $\mathrm{CR}$ at a structural brain level have also been investigated. Welborn et al. (2009), using tensor-based morphometry, investigated the relation between sex, emotionregulation abilities, and local brain volume of several frontal regions. The authors found that $\mathrm{CR}$ positively correlated with the vmPFC volume, whereas suppression was negatively correlated with vmPFC volume (Welborn et al., 2009). Giuliani et al. (2011) examined the relation between CR and the dorsal anterior cingulate cortex (dACC) volume in a non-clinical population. They focused their attention a priori on dACC based on its role in psychopathological conditions characterized by dysregulated emotions (Asami et al., 2008; Giuliani et al., 2011; Vasic et al., 2008). The authors included in the regions of interest the ventral anterior cingulate cortex (vACC) as a control region. Results showed a correlation between the volume of dACC and CR scores (ERQ). No relation was found between ERQ scores and VACC volume, suggesting a prominent role of dACC in CR (Giuliani et al., 2011). More recently, Hermann et al. (2014) found a positive correlation between $\mathrm{CR}$ and amygdala volume using voxel-based morphometry (Hermann et al., 2014).

Unfortunately, no coherent picture emerges from these studies. Some inconsistencies may be attributed to differences in the methodologies used and in the heterogeneity of the sample with respect to for example age, or may be due to the presence of diffuse structural abnormalities, which are hard to capture with a non-whole-brain approach. For example, voxel-based morphometry (VBM) and region-of-interest (ROI) analysis may both suffer from some limitations when studying complex and distributed functions such as emotionregulation abilities. Both VBM and ROI analyses look for focal differences in terms of gray matter in a priori chosen regions, ignoring the possibility that other regions may also be affected. It has been demonstrated that VBM, as a univariate approach, shows residual background noise and, more importantly, it does not provide information about the relationship between regions (Xu et al., 2009). This limits the interpretation of the results, particularly when investigating complex systems (see Pappaianni et al., 2018, for discussion). Contrary to VBM and ROI analysis, a whole-brain analysis does not impose an a priori selection of regions assumed to be involved in performing the task (as in ROI analysis), but it acts blindly on the whole brain, allowing for unanticipated patterns to also be detected.

\section{Psychological features associated with reappraisal}

In addition to the limited knowledge about the neural substrates of $\mathrm{CR}$, there is still a debate on psychological and affective effects related to CR. Although we know from several studies that reappraisal dampens task-related negative emotions (Grecucci et al., 2013a,b; Gruber et al., 2014; Winecoff et al., 2011), we still do not know how individual differences in the daily usage of CR are associated with the strength and valence of emotions. Meyer et al. (2012) tested 
the efficacy of reappraisal and suppression to regulate everyday affective experiences. A significant negative correlation was found between reappraisal and mean level of arousal, whereas no relation was found with valence of daily affective experiences. In addition, a negative correlation between reappraisal and positive as well as negative affectivity emerged (Meyer et al., 2012).

Andreotti et al. (2013) investigated the possible relation between reappraisal, working memory, positive and negative affect, and symptoms of anxiety and depression. In this correlational study, reappraisal emerged as positively correlated with the positive affect and negatively correlated both with the negative affect and with anxiety/depression symptoms (Andreotti et al., 2013), suggesting a main role of reappraisal in general psychophysical well-being that impacts both negative and positive affect. When assessed by an experimental task during fMRI, reappraisal showed a positive correlation with activation of the lateral and medial prefrontal cortices. Reappraisal also showed a negative correlation with traitanxiety symptoms, a positive correlation with the positive affect, and no correlation with negative affect (Uchida et al., 2015).

Gunaydin et al. (2016) showed that individual differences in reappraisal ability were a predictor of lower increases in negative affect against negative events, in addition to being a predictor of lower changes in positive affect responding to positive events in daily life.

This pattern is quite complex, indicating that the relationship between reappraisal and affectivity is still unclear and does not seem to be univocal. Specifically, the available empirical evidence does not disambiguate whether reappraisal acts by increasing the positive affectivity symptoms or by decreasing the negative ones. What is lacking in this literature is a direct comparison between the positive and negative affectivity of low versus high reappraisers: this comparison could better characterize the effects of usage of cognitive reappraisal in everyday life. One limitation of all the studies cited above is that they used correlational methods rather than a more easily interpretable two-samples design.

Besides affecting positive and negative affectivity, some emotion regulation strategies may also have an influence at the level of interpersonal abilities. Compared with expressive suppression, cognitive reappraisal requires less self-regulation effort acting immediately before the emotional experience, i.e., as antecedent-focused emotion (Gross, 2002). Starting from this, reappraisal may induce more prosocial behavior than suppression (Gross, 2002; Laghi et al., 2018). It follows that manifesting more social behavior involves opening oneself to others and to the mental states of others. Specifically, empathy is defined as the ability to understand and respond to the affective experience of other people (Decety \& Jackson, 2006; Laghi et al., 2018). Decety (2010) proposed a theoretical model of human empathy developing during childhood and adolescence that incorporates three components: affective arousal, the recognition of emotion, and the regulation of emotion. Emotion dysregulation may thus be triggered by a deficit in empathy. This claim has been put forward by Schipper and Petermann (2013), who argued that specific problems in the theory of mind and in taking the perspective of others may lead to deficits in reading and understanding one's own emotions and those of others, also leading to emotion-regulation problems (Schipper \& Petermann, 2013). Lockwood et al. (2014) explored this issue by testing a possible relation between emotional empathy, emotional regulation, and prosocial behavior. They found an effect of reappraisal as modulator of affective empathy and prosocial tendencies. Moreover, a person's low or average usage of reappraisal was correlated with empathy and prosocial behavior, whereas a high level of daily reappraisal was not. Lebowitz \& Dovidio (2015) showed that while empathic concern was negatively correlated with suppression, it was positively correlated with reappraisal. As recently argued by Laghi et al. (2018), while another regulation strategy such as suppression fails to modulate positive social behavior (Gross \& John, 2003; Srivastava et al., 2009), there is no clear and exhaustive experimental evidence about a relationship between reappraisal and social cognition. In their recent study, the authors investigated the relation between emotion regulation strategies, empathy, and social conduct in middle adolescents. In this correlational study, reappraisal was positively correlated with empathy but negatively correlated with internalizing and externalizing behaviors.

Based on this evidence, empathy may have a mediation role between emotion regulation and positive social behavior. However, the results reported are not fully consistent, and a direct comparison between proneness to empathy in people who apply more or less reappraisal, which could highlight the real impact of reappraisal on social behavior towards others, is lacking.

\section{Aim of the study}

The aim of the present study was threefold. Our first aim was to investigate possible differences in gray matter volume between low and high reappraisers by using a multivariate whole-brain approach known as source-based morphometry (SBM) (aim 1). SBM is a data-driven method based on Independent Component Analysis that extracts an independent pattern of covariation in gray matter (i.e., "structural networks") from the structural images of participants (Xu et al., 2009). The use of this novel approach allows scientists to investigate and characterize differences among individuals without the inclusion of a priori selected ROIs, correlations between neural and behavioral variables, or a priori univariate methods (Grecucci et al., 2016; Pappaianni et al., 2018; Xu et al., 2009). Since the issue of the interpretation of the correlations between behavioral and neural variables has already 
been addressed (see, e.g., Lindquist \& Gelman, 2009; Vul et al., 2009; Yarkoni, 2009), our intention was to take a step forward by using a fully automated and data-driven method to analyze data. In our case, this method allowed us to characterize differences between people who rarely apply reappraisal (low reappraisers) and those who apply it more frequently (high reappraisers). In addition to this, we aimed to look at the relationship among the detected networks in terms of structural network correlations. Specifically, we investigated if intra-correlations among the automatically detected networks may differ between low and high reappraisers. Our prediction was that the frequency of application of reappraisal may affect the general configuration of networks correlations in the brain.

Second, we aimed at exploring psychological differences in terms of experienced positive and negative affect between low and high reappraisers (aim 2). To this end, we use the Positive and Negative Affect Schedule (PANAS; Watson et al., 1988) to measure the current affective state in both positive and negative terms.

Our third aim was to detect possible differences between reappraisers with respect to the tendency to display prosocial behavior (aim 3), as indexed by the Interpersonal Reactivity Index (IRI; Davis, 1980). We predicted that daily frequency of reappraising emotional events may have an impact on both affectivity and sociality.

\section{Method}

\section{Participants and behavioral testing}

Forty healthy participants were recruited from the local population of Trentino-Alto Adige (Italy). They provided informed consent to participate in this study, according to guidelines set by the Ethics Committee of the University of Trento.

T1-weighted anatomic images were acquired using a 4T Bruker MedSpec Biospin MR scanner and a birdcage transmit, eight-channel receiver head radiofrequency coil (MP-RAGE; $1 \times$ $1 \times 1 \mathrm{~mm}^{3}$; FOV, $256 \times 224 \mathrm{~mm}^{2}$; 176 slices; GRAPPA acquisition with an acceleration factor of 2; TR, 2,700 ms; TE, 4.18 $\mathrm{ms}$; inversion time (TI), 1,020 ms; $7^{\circ}$ flip angle). Because of corrupted unusable anatomic data, three participants were excluded from the analysis. Outside of the scanner, participants completed the Italian version (Balzarotti et al., 2010) of the Emotion Regulation Questionnaire (ERQ; Gross \& John, 2003), a 10-item scale designed to measure respondents' frequency of usage of two emotion-regulation strategies: cognitive reappraisal (CR) and expressive suppression. In the scale, each item is associated with a 7-point Likert-type scale ranging from 1 (strongly disagree) to 7 (strongly agree). Participants had to indicate their degree of agreement on each item, i.e., sentences regarding different ways of using the two strategies in everyday life.
In order to investigate the participants' current affect, the Italian version (Terracciano et al., 2003) of the PANAS (Watson et al., 1988) was used. PANAS is a 20 -word-item questionnaire describing emotional state: participants need to indicate on a 5-point Likert scale how that emotional state was representative of their current internal situation, ranging from "Very slightly or not at all (1)" to "Extremely (5)."

Finally, participants completed a reduced version of the Interpersonal Reactivity Index (IRI; Davis, 1980). IRI is a 28-item questionnaire answered on a 5-point Likert scale ranging from "Does not describe me well (1)" to "Describes me very well (5)." In the classical version, four subscales are derivable (each one composed of seven items): Perspective Taking (PT), Fantasy (FA), Empathic Concern (EC), and Personal Distress (PD). Since our research interest was predominantly for empathy and sociality, we used the Italian version (Albiero et al., 2006) with 14 items referring to the PT and EC subscales.

Since our intention was to explore whether the frequency of applying reappraisal may leave a structural trace in the brain and have an impact on social-affective traits, we split participants into two subgroups based on the median $($ median $=27)$ of the scores of the ERQ-Reappraisal subscale (see Table 1 for details). Participants with a CR score below 27 entered the group of low reappraisers $(n=18)$, whereas participants with a CR score higher than 27 entered the high reappraisers group $(n=19)$. Demographic information is summarized in Table 1 .

\section{MRI data processing}

During the initial scrupulous quality check of the MRI data, three participants were excluded from the analysis because of artifacts in the $\mathrm{T} 1$ or corrupted data (one from the group of low reappraisers and two from the group of high reappraisers). Preprocessing of the images was performed using the Computational Anatomy Toolbox (CAT12, http://www. neuro.uni-jena.de/cat/), a toolbox for SPM12 software (http://www.fil.ion.ucl.ac.uk/spm/software) working in the Matlab environment (The Mathworks, Natick, MA, USA). After setting the origin, the $37 \mathrm{~T} 1$-weighted images were segmented in gray matter, white matter, and cerebrospinal fluid. Since we were only interested in gray matter, the modulated images of gray matter were normalized to MNI space with spatial smoothing (full-width at half maximum of Gaussian smoothing kernel $[8,8,8])$ in SPM12. The Group ICA of an FMRI Toolbox (GIFT, http://mialab.mrn.org/software/gift/) was used to perform SBM. SBM is a whole-brain data-driven multivariate approach based on the Independent Component Analysis (ICA) (Lee et al., 1999; Xu et al., 2009). ICA detects and reconstructs the signal coming from different sources in the gray matter images, identifying independent clusters of voxels covariant along the entire sample (Xu et al., 2009). These sets of voxels are considered to be independent gray 
Table 1 Age, Education and ERQ-Reappraisal scale scores among low and high reappraisers. Group, numerosity (N), mean, standard deviation (SD) for each variable are shown, in addition to Student' t, $\mathrm{p}$ value and Cohen's $\mathrm{d}$ for each comparison between groups

\begin{tabular}{llllll}
\hline & Group & $N$ & Mean & SD & Mean difference \\
\hline Age & Low reap. & 18 & 23.17 & 3.485 & $\mathrm{t}(35)=-0.878, \mathrm{p}=0.386$ \\
& High reap. & 19 & 24.21 & 3.735 & $\mathrm{~d}=-0.289$ \\
Education & Low reap. & 18 & 15.56 & 2.287 & $\mathrm{t}(35)=-1.126, \mathrm{p}=0.268$ \\
& High reap. & 19 & 16.34 & 1.958 & $\mathrm{~d}=-0.370$ \\
ERQ-Cognitive Reappraisal & Low reap. & 18 & 21.44 & 3.944 & $\mathrm{t}(35)=-8.093, \mathrm{p}=<.001$ \\
& High reap. & 19 & 32.26 & 4.175 & $\mathrm{~d}=-2.662$ \\
\hline
\end{tabular}

matter networks. In our case, the extraction of 20 independent components was called for, as indicated by default in GIFT. Infomax, a neural network algorithm, was used to perform ICA in order to maximize the recognition of independent components from the images (Bell \& Sejnowski, 1995; Lee et al., 1999). As the stability analysis type, ICASSO (http:// research.ics.aalto.fi/ica/icasso/) with both Bootstrap and RandInit was chosen for investigating the reliability of the ICA algorithm. ICA was run 100 times: the minimum cluster size was fixed at $80(0.8 *$ number of ICA runs), the maximum at 100 (equal to the number of runs). Minimum and maximum cluster sizes were set up based on SBM's manual suggestions. SBM transforms gray matter volumes into a numerical vector: the output of SBM is a numerical coefficients matrix, composed of rows (participants) and columns (the extracted components). In other words, for every participant, a loading numerical coefficient shows how much a component is expressed in her/him (Pappaianni et al., 2018).

In addition, in order to explore the relationship between the components, Structural Networks Correlations (SNC) analysis was computed for both low and high reappraisers between the spatial-loading coefficients of every component (Segall et al., 2012). To examine differences in the network matrices R1 and R2 for g1 (low reappraisers) and g2 (high reappraisers) groups, we adopted a non-parametric bootstrap significance test (Hall \& Wilson, 1991) and a hierarchical clustering. The bootstrap analysis provided results to assess whether R1 = R2 in the two groups. By contrast, clustering yielded results about structural differences eventually present in the networks R1 and R2. The similarity of R1 and R2 was assessed through Bhattacharyya statistics (Victor \& Ricardo, 1990) defined for two Gaussian-distributed samples:

$\delta\left(R_{1}, R_{1}\right)=\frac{1}{2} \log \left(\frac{0.5 \operatorname{det}\left(R_{1},+R_{1}\right)}{\sqrt{\operatorname{det}\left(R_{1}\right)+\operatorname{det}\left(R_{2}\right)}}\right)$

The resampling study was designed in order to determine the distribution $\mathrm{F}(\delta(\mathrm{R} 1, \mathrm{R} 2) \mid \mathrm{H} 0)$ under the hypothesis $H 0: R 1=R 2$. The study involved $M=25,000$ resamples from $\mathrm{g} 1$ and $\mathrm{g} 2$ with sizes equal to $\mathrm{Ng} 1$ and $\mathrm{Ng} 2$. For each pair of samples, we computed the distance in the previous equation. The hypothesis H0: R1 = R2 was evaluated by computing the p-value

$P\left(\delta^{1: M}>\delta^{*}\left(R_{1}, R_{2}\right) H_{0}\right)$

on the bootstrap distribution, with $\delta *(\mathrm{R} 1, \mathrm{R} 2)$ being the observed statistics obtained on the empirical samples. Eventual structural differences regarding R1 and R2 were evaluated by means of a hierarchical Wald's clustering applied on the distance-based transformations of $\mathrm{R} 1$ and R2.

\section{Results}

\section{Morphometric analysis}

SBM returned 20 independent components. Specifically, a matrix of loading coefficients was obtained, composed of 37 rows (equal to the number of participants) and 20 columns (independent components). SBM was run considering all the participants together, separating the two groups only a posteriori. So, based on the reappraisal score, the matrix was divided into two sub-matrices representing the low and high reappraisers, respectively, with 18 and 19 rows and with 20 columns each. An independent-samples Welch t-test on the matrix's loading coefficients was used to investigate differences between low and high reappraisers for each component. Independent Component 16 was statistically significant $(\mathrm{t}(34.17)=2.618, \mathrm{p}$ $=0.013$, Cohen's $\mathrm{d}=0.859$ ) among reappraisers. Loading coefficient's distribution boxplot of Independent Component 16 is available for each group in Fig. 1b. Details for each component are available in the Supplementary Materials. Independent Component 16 (IC16) is a wide component that included, among others, clusters of voxels in the bilateral Inferior Frontal Gyrus, Middle Temporal Gyrus, Middle Frontal Gyrus, Inferior Parietal Lobule, Precuneus, Cuneus, right Postcentral and Precentral Gyrus, Culmen, left InferiorMiddle Occipital Gyrus. Details of IC16 are available in Table 2, while a visual rendering is shown in Fig. 1a. 


\section{Structural networks correlations}

Conditioned on the sample sizes $\mathrm{Ng} 1$ and $\mathrm{Ng} 2$ with $\alpha=5 \%$, a significant difference among g1 and g2 emerged in terms of R1 and R2 (Fig. 1 in the Supplementary Materials). Structural differences regarding $\mathrm{R} 1$ and $\mathrm{R} 2$ were evaluated by means of a hierarchical Wald's clustering applied on the distance-based transformations of R1 and R2. Figures 2, 3, 4, and 5 in the Supplementary Material show the results for the network matrix R1 (low reappraisers). This method shows how variables are grouped in two main clusters (c1 and $\mathrm{c} 2$ ) with two further sub-clusters (c11, c12 and c21, c22). This result was assessed using the common Silhouette index (Arbelaitz et al., 2013), which showed the highest value for the two-cluster solution (Fig. 2 in the Supplementary Material). As for the network matrix R2 (high reappraisers), two clusters were found to summarize all the variables involved (Figs. $2 b-5 b$ in the Supplementary Material). This was also confirmed by the Silhouette index (Fig. 2b). Clusters are as follows:

$$
\begin{gathered}
c_{1}=\{\underbrace{\left\{X_{19}, X_{3}, X_{7}, X_{6}, X_{4}, X_{1}\right\}}_{c_{11}}, \underbrace{\left\{X_{13}, X_{12}, X_{10}\right\}}_{c_{12}}\} \\
c_{2}=\{\underbrace{\left\{X_{11}, X_{8}, X_{17}, X_{20}, X_{5}\right\}}_{c_{21}}, \underbrace{\left.\left\{X_{16}, X_{18}, X_{2}, X_{15}, X_{9}, X_{14}\right\}_{c_{22}}\right\}}_{c_{22}}
\end{gathered}
$$

We further investigated how the dendrograms of low (Fig. 1, Supplementary Material) and high reappraisers (Fig. 1b, Supplementary Material) overlap. This has been assessed using the tanglegram graph (see Fig. 1e) and two measures, the entanglement coefficient and the Baker's gamma correlation coefficient (Baker, 1974). Lower values for the entanglement coefficient indicate the dendrograms are very similar, whereas high values for the coefficient mean the dendrograms differ sufficiently. By contrast, Baker's correlation is interpreted as the standard correlation coefficient. These tools were used as implemented in the R package "dendextend" (Galili, 2015). The results in Fig. 1c indicate that - although clustering solutions are the same for both R1 and R2 - the two dendrograms are quite different as the straight lines among the nodes are mixed up. The entangle coefficient is quite large $(=0.92)$, whereas Baker's correlation is low $(=0.37)$.

In conclusion, the analysis proposes that R1 and R2 for high and low reappraisers are different in terms of distance (bootstrap test). The clustering analysis suggests structural differences between the two network matrices. In particular, both the networks show the same clustering solutions (two main clusters, see Silhouette index) but they map the 20 ICA components involved differently. Moreover, the structures of the networks seem to be different overall in terms of similarity, as shown by the high entangle coefficient and low Baker's correlation.

\section{Behavioral results}

Statistical analysis of behavioral data was done using JASP v. 0.8.5.1 (https://jasp-stats.org/) and Jamovi v. 0.9.5.13 (https:// www.jamovi.org/). Tests of Normality of Shapiro-Wilk and Test of Equality of Variances (Levene's) resulted in being not significant for each variable, suggesting a normal distribution of the data and equal variances between the two groups. A two-sample t-test assuming equal variance was used on behavioral data in order to find differences between low and high reappraisers. A significant difference emerged in the Negative Affectivity subscale of PANAS (PANAS-NA) $(\mathrm{t}(35)=2.133$, $\mathrm{p}$ $=0.04$, Cohen's $d=0.702$ ), suggesting a higher negative affectivity in low reappraisers (Fig. 1c). No other significant between-group differences were found for age, education, PANAS-PA, ERQ suppression, IRI-PT and IRI-EC (each $p$ > 0.05). Details are shown in Table 3.

\section{Discussion}

In the present study, we aimed to elucidate whether the frequency of usage of reappraisal may be related to brain and psychological differences in the healthy population. We applied SBM, a wholebrain data-driven approach, to structural images of 37 participants without a history of psychiatric or neurological problems in order to investigate large-scale gray matter differences between low and high reappraisers. SBM returned 20 different sources of signal. Among these, only the IC16 resulted in statistically significant differences between reappraisers, indicating a higher concentration of gray matter in specific brain regions in low reappraisers. This component revealed an increased gray matter concentration in a network including a large cluster in the frontal and temporal lobes, but also in occipital-parietal regions and in a small portion of the anterior cerebellum. Our interpretation is that an increase of gray matter in specific regions involved in emotion regulation may be strictly related to anomalous functioning of those areas. Such abnormal structural and functional increases in populations characterized by psychiatric disorders are not new in the literature; however, these differences have not been previously shown in the normal population. Below we review our findings by making comparisons with the existing literature. 

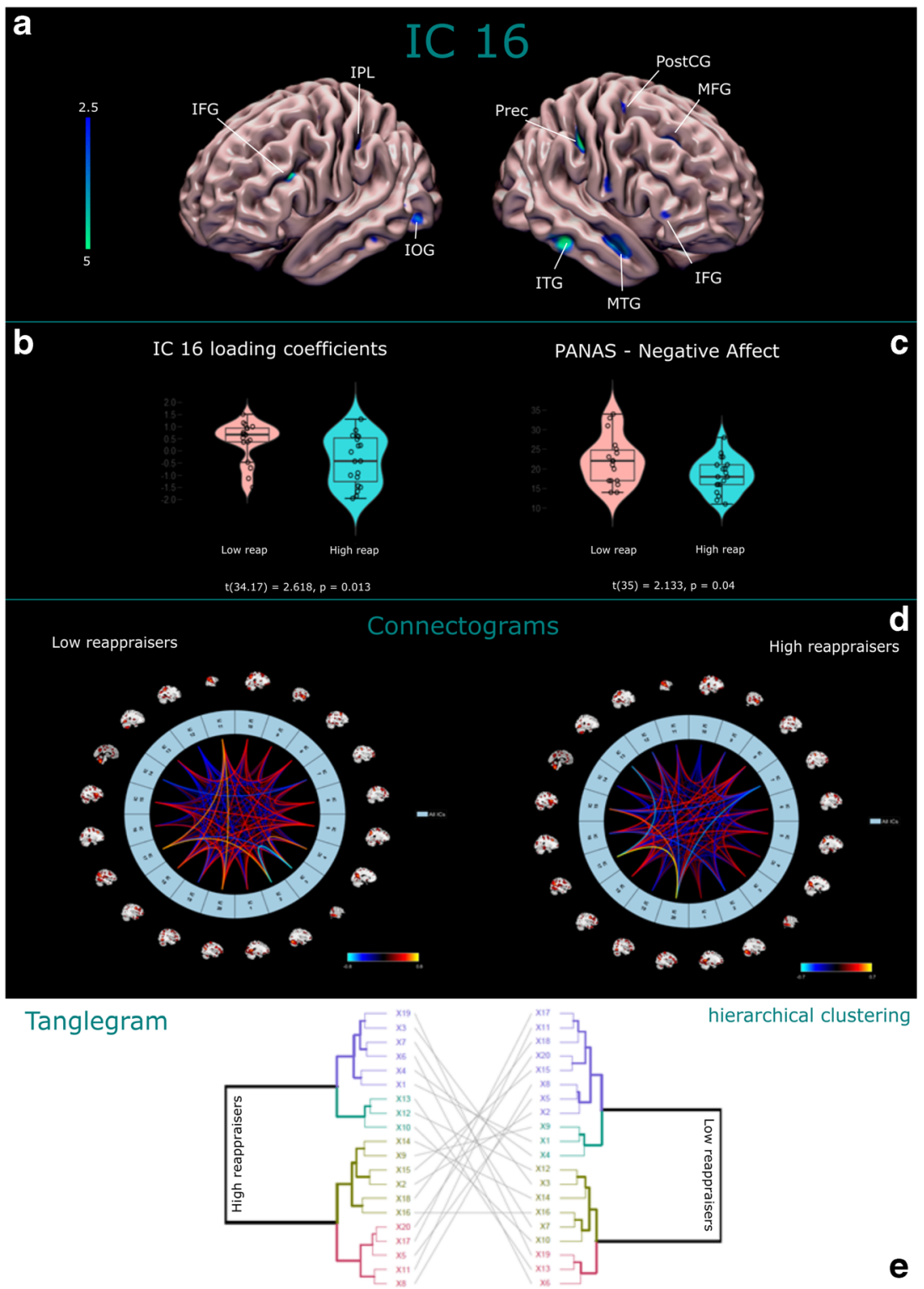

Fig. 1 a-b) Visual rendering of IC16 and IC16 loading coefficients distribution between groups. c) Violin boxplots of PANAS-NA scores distribution among low and high reappraisers; c) Connectograms of the structural network correlations matrices for low and high reappraisers; d)

Within IC16, a significant cluster was found in the bilateral inferior frontal gyrus (IFG), with a slight prevalence in the right hemisphere. It is known that frontal areas play a decisive role in top-down emotion regulation (Grecucci et al., 2013a,b). Using a fear-conditioning task, Cha et al. (2016) recently investigated the role of IFG in patients with general anxiety disorder (GAD). As with any anxiety disorder, GAD is characterized by a strong emotional dysregulation, which creates a strong experience of fear and discomfort in response to a salient elicitor in the present. The authors found that GAD patients exhibited anomalous functional and structural prefrontal-limbic connectivity (comprising the IFG, vmPFC, and amygdala). While the IFG was
Tanglegram between the dendrograms of R1 (high reappraisers, on the left) and R2 (low reappraisers, on the right). Note that the lines among nodes indicate how much the dendrograms entangle

connected with the vmPFC, no direct IFG inhibitory effects on the amygdala were reported (as occurred between the vmPFC and amygdala). For this reason, they suggested that the IFG inhibits the amygdala only through the vmPFC in GAD patients, impacting in this way their emotional regulation difficulties.

Medial prefrontal regions seem to also be decisive in rumination, or thinking around a common instrumental theme that takes place in the absence of immediate environmental demands (Martin \& Tesser, 1996; p. 7). Rumination typically occurs in individuals suffering from anxiety and depression (Aker et al., 2014; Cooney et al., 2010; Nolen-Hoeksema et al., 2008). An increase in neural activity was detected in 
Table 2 Independent Component 16. Talairach labels of regions of interest, Brodmann area, volume (expressed in cc) and spatial MNI coordinates are shown

\begin{tabular}{llll}
\hline Area & Brodmann Area & volume $(\mathrm{L} / \mathrm{R})$ & $\mathrm{MNI}(\mathrm{x}, \mathrm{y}, \mathrm{z})$ \\
\hline Inferior Frontal Gyrus & 44,8 & $1.3 / 1.5$ & $(-39,19.5,25.5) /(24,24,42)$ \\
Middle Temporal Gyrus & $20,21,39$ & $0.1 / 1.7$ & $(-51,-57,45) /(60,-34.5,-16.5)$ \\
Inferior Occipital Gyrus & 19 & $0.3 / 0.0$ & $(-40.5,-70.5,-10.5) /-$ \\
Postcentral Gyrus & $2,3,40$ & $0.0 / 0.8$ & $-/(39,-30,42)$ \\
Inferior Temporal Gyrus & 20 & $0.0 / 1.0$ & $-/(57,-34.5,-19.5)$ \\
Middle Frontal Gyrus & 8,46 & $0.2 / 0.6$ & $(-42,19.5,28.5) /(24,21,45)$ \\
Middle Occipital Gyrus & $*$ & $0.3 / 0.0$ & $(-40.5,-73.5,-13.5) /-$ \\
Angular Gyrus & $*$ & $0.1 / 0.0$ & $(-31.5,-61.5,36) /-$ \\
Inferior Parietal Lobule & 40 & $0.4 / 0.1$ & $(-34.5,-61.5,39) /(39,-34.5,43.5)$ \\
Fusiform Gyrus & 20 & $0.0 / 0.1$ & $-/(54,-31.5,-25.5)$ \\
Precuneus & $*$ & $0.1 / 0.1$ & $(-28.5,-61.5,39) /(16.5,-60,31.5)$ \\
Culmen & $*$ & $0.0 / 0.5$ & $-/(16.5,-39,-21)$ \\
Cuneus & 18 & $0.2 / 0.1$ & $(-19.5,-84,22.5) /(6,-79.5,21)$ \\
Precentral Gyrus & 6 & $0.0 / 0.3$ & $-/(58.5,-15,19.5)$ \\
Inferior Frontal Gyrus & $*$ & $0.0 / 0.1$ & $-/(51,33,15)$ \\
\hline
\end{tabular}

the dIPFC, OFC, and subgenual ACC in patients with depression during rumination (Cooney et al., 2010). A review article on neuroimaging studies reported an anomalous increased functional activity in patients with major depressive disorder during voluntary control of emotion regulation within the lateral prefrontal cortices (Rive et al., 2013). Kühn et al. (2014) investigated the neural bases of unwanted thoughts, which have a significant impact on the emotional and psychophysical well-being of individuals. Studying the functional connectivity during resting-state fMRI, they found higher connectivity between the left putamen and the left IFG in people with more unwanted thoughts, in addition to a relation between increases in unwanted thought states and lower local

Table 3 PANAS-PA (Positive Affectivity), PANAS-NA (Negative Affectivity), PANAS-Total score, ERQ-Expressive Suppression, IRIPerspective Taking, IRI-Empathic Concern subscales among low and connectivity in the right dIPFC (Kühn et al., 2014). Another case in point is borderline personality disorder (BPD). From a structural point of view, BPD seems to be characterized by abnormalities in gray matter volume, particularly in the amygdala and hippocampus (for a meta-analysis, see Nunes et al., 2009). From a functional point of view, BPD seems to be related to abnormal activity in a fronto-limbic network that includes mainly subcortical regions such as the amygdala and insula, respectively, with the anterior cingulate cortex (ACC), medial frontal cortex, orbitofrontal cortex (OFC) and dIPFC, which are regions important for inhibition-regulatory processes (Krause-Utz et al., 2014). The authors proposed that the impulsivity and interpersonal symptoms characterizing BPD

\begin{tabular}{|c|c|c|c|c|c|}
\hline & Group & $N$ & Mean & $S D$ & Mean difference \\
\hline \multirow[t]{2}{*}{ PANAS-PA (Positive Affectivity) } & Low reap. & 18 & 32.72 & 5.188 & \multirow[t]{2}{*}{$\mathrm{t}(35)=-1.625, \mathrm{p}=0.113, \mathrm{~d}=-0.534$} \\
\hline & High reap. & 19 & 35.26 & 4.306 & \\
\hline \multirow[t]{2}{*}{ PANAS-NA (Negative Affectivity) } & Low reap. & 18 & 22.11 & 6.038 & \multirow[t]{2}{*}{$\mathrm{t}(35)=2.133, \mathrm{p}=0.040, \mathrm{~d}=0.702$} \\
\hline & High reap. & 19 & 18.42 & 4.401 & \\
\hline \multirow[t]{2}{*}{ PANAS Total score } & Low reap. & 18 & 54.83 & 8.104 & \multirow[t]{2}{*}{$\mathrm{t}(35)=0.470, \mathrm{p}=0.641, \mathrm{~d}=0.155$} \\
\hline & High reap. & 19 & 53.68 & 6.725 & \\
\hline \multirow[t]{2}{*}{ ERQ Expressive Suppression } & Low reap. & 18 & 12.44 & 5.170 & \multirow[t]{2}{*}{$\mathrm{t}(35)=-0.581, \mathrm{p}=0.565, \mathrm{~d}=-0.191$} \\
\hline & High reap. & 19 & 13.37 & 4.500 & \\
\hline \multirow[t]{2}{*}{ IRI Perspective Taking } & Low reap. & 18 & 22.61 & 4.075 & \multirow[t]{2}{*}{$\mathrm{t}(35)=-1.739, \mathrm{p}=0.091, \mathrm{~d}=-0.572$} \\
\hline & High reap. & 19 & 24.63 & 2.929 & \\
\hline \multirow[t]{2}{*}{ IRI Empathic Concern } & Low reap. & 18 & 24.11 & 3.771 & \multirow[t]{2}{*}{$\mathrm{t}(35)=-0.693, \mathrm{p}=0.493, \mathrm{~d}=-0.228$} \\
\hline & High reap. & 19 & 24.95 & 3.566 & \\
\hline
\end{tabular}


patients may be the reflection of hyperactivity in the limbic circuit caused by an abnormal inhibitory effect of frontal regions (Krause-Utz et al., 2014). O'Neill and colleagues (2015) investigated functional connectivity in patients with BPD: during resting-state fMRI, an increase in functional connectivity between the precuneus, the left inferior frontal lobe, left precentral/middle frontal, and left middle occipital/superior parietal lobes emerged in patients with BPD. Notably, most of these regions are included in our network. The authors suggested that this increased connectivity may mirror ruminative thinking and continuous self-referential information, which characterize this personality disorder (O'Neill et al., 2015). Another study reported an increase in functional activity in the precuneus in patients with BPD during the resting state (Visintin et al., 2016), suggesting a critical role of this region in the disorder.

A recent meta-analysis reported hyperactivation of the bilateral dIPFC, the amygdala, and the posterior cingulate cortex (PCC) in patients with BPD during the elaboration of negative emotional stimuli, confirming the key role of these areas for emotion processing (Schulze et al., 2016). During an emotioninducing task in an fMRI scanner, at the sight of pictures with negative emotional content, patients with BPD showed an increase in activation in the amygdala, fusiform gyrus, primary visual areas, superior temporal gyrus, and premotor areas (Koeningsberg et al., 2009). Beyond the superior temporal gyrus and the amygdala, whose critical role in the encoding of emotional stimuli is widely recognized, the fusiform gyrus, with primary visual areas and premotor areas, were included in our circuit, suggesting a possible implication of these regions in anomalous elaboration of emotion, as in BPD. All these studies point toward the idea that structural and functional increases in these areas may lead to anxiety, depression, impulsivity, and emotion dysregulation. These results are in line with our main findings of increased gray matter concentration (IC16) in individuals characterized by a low reappraisal usage.

Another region that emerged in our analysis was the inferior parietal lobule (IPL). The involvement of parietal regions is not new in emotion regulation. A closer look to the neural substrates of emotion regulation suggests that temporal inferior parietal in addition to ventromedial prefrontal regions are involved in the application of emotion regulation strategies (Buhle et al., 2014). These areas fall into what is called the semantic system, which is critical in high-level integrative processes (Binder et al., 2009; Patterson et al., 2007). A recent meta-analysis proposes a possible integration between emotion regulation and the semantic system, suggesting that executive functions (guided by frontal regions) are just one factor in emotion regulation, while semantic encoding may be another important factor (Messina et al., 2015). As far as our results are concerned, we can assume that an overload of gray matter in parietal (such as IPL) and temporal regions can destabilize the semantic network at a functional level, not correctly managing the semantic information and making the application of the strategy more difficult. IC16 also includes the temporoparietal junction (TPJ). It has been widely shown that the TPJ is a key region for the theory of mind and for the understanding of other people's beliefs (Gallagher et al., 2000; Saxe \& Kanwisher, 2003). Studies of patients with lesions in the left TPJ have provided further evidence of a direct involvement of this region in invoking someone's beliefs (Samson et al., 2004). Understanding mental states is a determining factor in the construction of effective social relationships, and since reappraisal encompasses the ability to see reality from another's point of view, anomalous functioning of the TPJ guided by an overload of gray matter may have an impact on the ability to reinterpret reality in a more efficient way.

Another region included in IC16 is the right fusiform gyrus $(\mathrm{rFG})$. The $\mathrm{rFG}$ has always been recognized as a crucial center for the processing of social stimuli such as faces (Frick et al., 2013; Haxby et al., 2000; Haxby et al., 2002). Frick et al. (2013) investigated the role of the fusiform gyrus in patients with social anxiety disorder (SAD), a disorder characterized by fear and avoidance of social situations, distorted negative self-beliefs (Goldin et al., 2009), and a maladaptive application of emotion-regulation strategy. Patients with SAD showed hyperreactivity in the bilateral fusiform gyrus responding to fearful faces. By taking into account the fusiform gyrus in a functional connectivity analysis, they found a greater connectivity between this region and the amygdala as well as a weaker connectivity with the vmPFC (Frick et al., 2013). In an implicit emotionprocessing task, a stronger activation of the right fusiform gyrus was found in patients with anorexia nervosa in response to all facial expressions, suggesting an anomalous implicit emotion elaboration during the precocious perceptual processing of the social stimulus (Fonville et al., 2013).

To our knowledge, there is no evidence about a direct involvement of the primary somatosensory areas (as post-central gyrus) in the regulation of emotions that we found partially included in IC16. Sensorimotor cortices seemed to be related to pain in association with emotional stimuli like facial expressions: in a MEG study, faces with emotional expressions and faces with neutral expressions were accompanied by pain evoked by electric stimulation. Emotional faces led to a stronger suppression of the pain-induced beta-band activity $(15-25 \mathrm{~Hz})$ in the aforementioned cortices, suggesting the activation of the sensorimotor system (Senkowski et al., 2011). Therefore, according to the authors, facial expression stimuli may impact the processing of acute pain. However, given the scarcity of evidence, it is not possible to provide a clear justification for our results. Future studies will need to understand if such areas can have a significant impact on the ability to regulate emotions at a cognitive level. We suggest that abnormalities in such structures could modulate the perception of pain occurring during the interaction with emotional stimuli.

In order to understand the relationship among the ICs extracted by SBM and to test for possible differences between the two populations, we calculated the statistical correlation between all the ICs in the two groups. We compared the two 
matrices obtained via a bootstrap procedure and hierarchical clustering. Analyses showed that low and high reappraiser matrices were different in terms of distance, a parameter suggestive of how the ICs are related to each other. Although ICs were assembled into two main clusters in both groups, the structures of the networks are assembled differently, indicating dissimilarity between correlations of structural networks in low and high reappraisers (Fig. 1). Specifically, IC16 is less related to other components in the low reappraisers. Our explanation is that in low reappraisers IC16 is more "isolated" from the other 19 circuits, whereas in high reappraisers IC16 interacts better with the other networks.

From a psychological point of view, our results showed that people characterized by low reappraisal usage seem more prone to greater negative affect. Clearly, it is not possible to infer that a non-adaptive regulation of emotions leads to a more negative perceived affectivity, nor can the contrary be demonstrated. It is known that negative affect consists of a general subjective distress related to aversive mood states, such as guilt, anger, fear, and nervousness (Miller et al., 2012; Watson et al., 1988). What our data suggest is that low frequency of usage of reappraisal, a strategy globally recognized as adaptive, is associated with a more negative affect.

No apparent relationship between cognitive reinterpretation abilities and empathy was found. This seems contradictory to the literature: as mentioned in the introduction, empathy has been found to have a direct influence on emotional regulation and individual well-being (Decety, 2010; Laghi et al., 2018; Lebowitz \& Dovidio, 2015; Lockwood et al., 2014). Nonetheless, it is interesting to note that the cerebral bases of empathy found in the literature underline many regions included in the IC16 network. Shamay-Tsoory (2011) stated that the inferior frontal gyrus and the inferior parietal lobule are necessary for emotion recognition and emotional contagion, while the vmPFC, the TPJ, and the medial temporal lobe are important for cognitive empathy (Shamay-Tsoory, 2011). Another study investigated the relation between gray matter volume and empathy traits: the left precuneus, inferior frontal gyrus, and anterior cingulate emerged as negatively correlated with the IRI's EC subscale, while PT abilities resulted in positive correlations with gray matter volume in the anterior cingulate (Banissy et al., 2012). To summarize, as the precuneus and the inferior frontal gyrus emerged in our network, we do not exclude that this circuit may also impact the empathic abilities of reappraisers, and thus be related to the ability to reinterpret reality.

\section{Conclusions}

The present study was specifically designed to show possible psychological and neural differences between low and high reappraisers by capitalizing on a multivariate analysis known as source-based morphometry. The circuit found to be different between the two groups included regions throughout the brain, suggesting that the ability to reappraise involves multiple regions. From a psychological point of view, low reappraisers showed higher negative affectivity, confirming the association between dysfunctional emotion regulation and affectivity.

However, this study has several limitations. First of all, the three questionnaires that we used (ERQ, PANAS, and IRI) are all self-report questionnaires, and biases associated with this type of tools are not rare and can have an impact on the investigated dimension (Cook, 2010). Unfortunately, three subjects were rejected because of artifacts in the images, limiting the experimental group to 37 participants. Based on the literature, this number of participants seemed adequate, although a larger number would always be desirable.

That said, this study provides the first evidence of differences in gray matter between low and high reappraisers in a whole-brain analysis. With further explorations, our results may have an impact in the emotion regulation field by characterizing individual differences in terms of frequency of usage of emotion regulation strategies.

Open Practices Statement Neural and behavioral data will be made available on request.

Author contributions EP and AG contributed to the experimental design, data collection, data analysis, and writing the paper; NDP contributed to data collection and writing the paper; RS and AC contributed to data analysis and writing the paper; $\mathrm{CC}$ and $\mathrm{RJ}$ contributed to writing the paper.

\section{Compliance with ethical standards}

Conflict of interest The authors declare that this study was conducted in the absence of any commercial or financial relationship that could be construed as a potential conflict of interest.

\section{References}

Aker M., Harmer C., Landrø N.I. (2014) More rumination and less effective emotion regulation in previously depressed women with preserved executive functions. BMC Psychiatry. 2014 Nov 27;14:334

Albiero, P., Ingloglia, S., Lo Coco, A. (2006). Contributo all'adattamento italiano dell'Interpersonal Reactivity Index di Davis [A contribution to the Italian validation of the Interpersonal Reactivity Index]. TPM, $2,107-12$

Andreotti, C., Thigpen, J.E., Dunn, M.J., Watson, K., Potts, J., Reising, M.M., Robinson, K.E., Rodriguez, E.M., Roubinov, D., Luecken, L., Compas, B.E. (2013) Cognitive reappraisal and secondary control coping: associations with working memory, positive and negative affect, and symptoms of anxiety/depression, Anxiety, Stress, \& Coping: An International Journal, 26:1, 20-35

Arbelaitz, O., Gurrutxaga, I., Muguerza, J., PeRez, J. M., Perona, I. (2013). An extensive comparative study of cluster validity indices, " Pattern Recognition, vol. 46, no. 1, pp. 243-256.

Baker, F. B. (1974). Stability of two hierarchical grouping techniques case i: Sensitivity to data errors. Journal of the American Statistical Association, vol. 69, no. 346, pp. 440-445. 
Balzarotti, S., John, O.P., Gross, J.J. (2010) An Italian adaptation of the Emotion Regulation Questionnaire. European Journal of Psychological Assessment, 26(1), 61-67

Banissy, M.J., Kanai, R., Walsh, V., Rees, G. (2012) Inter-individual differences in empathy are reflected in human brain structure. NeuroImage 62 (2012) 2034-2039

Banks, S.J., Kamryn, T.E., Angstadt, M., Nathan, P.J., Phan, K.L. (2007) Amygdala-frontal connectivity during emotion regulation. Soc Cogn Affect Neurosci. 2007 Dec; 2(4): 303-312.

Binder, J. R., Desai, R. H., Graves, W. W., Conant, L. L. (2009). Where is the semantic system? A critical review and meta-analysis of 120 functional neuroimaging studies. Cereb. Cortex 9, 2767-2796.

Buhle J.T., Silvers J.A., Wager T.D., Lopez R., Onyemekwu C., Kober H., Weber J., Ochsner K.N. (2014) Cognitive reappraisal of emotion: a meta-analysis of human neuroimaging studies. Cereb Cortex. 2014 Nov; 24(11):2981-90

Burklund, L., Creswell, J.D., Irwin, M.R., Lieberman, M.D. (2014) The common and distinct neural bases of affect labeling and reappraisal in healthy adults. Front Psychol. 2014 Mar 24;5:221. doi: https:// doi.org/10.3389/fpsyg.2014.00221.

Carlson, J. M., \& Mujica-Parodi, L. R. (2010). A disposition to reappraise decreases anterior insula reactivity during anxious anticipation. Biol. Psychol. 85, 383-385. doi: https://doi.org/10.1016/j.biopsycho. 2010.08.010

Cha, J., DeDora, D., Nedic, S., Ide, J., Greenberg, T., Hajcak, G., MujicaParodi, L.R. (2016) Clinically Anxious Individuals Show Disrupted Feedback between Inferior Frontal Gyrus and Prefrontal-Limbic Control Circuit. The Journal of Neuroscience, April 27, 2016. 36(17): $4708-4718$

Cook, C. (2010) Mode of administration bias. J Man Manip Ther. 2010 Jun; 18(2): 61-63.

Cooney, R.E., Joormann, J., Eugène, F., Dennis, E.L., Gotlib, I.H. (2010) Neural correlates of rumination and depression. Cogn Affect Behav Neurosci; 10(4): 470-478.

Cutuli, D. (2014). Cognitive reappraisal and expressive suppression strategies role in the emotion regulation: an overview on their modulatory effects and neural correlates. Front Syst Neurosci. 2014 Sep 19; 8:175. doi: https://doi.org/10.3389/fnsys.2014.00175.

Dadomo, H., Panzeri, M. Caponcello, D., Carmelita, A., Grecucci, A., (2018) Schema therapy for emotional dysregulation in personality disorders: a review. Current Opinion in Psychiatry, 31(1), 43-49.

Davis, M.H. (1980). A multidimensional approach to individual differences in empathy. JSAS Catalog of Selected Documents in Psychology, 10, 85.

De Panfilis, C., Schito, G., Generali, I., Gozzi, L. A., Ossola, P., Marchesi, C., Grecucci, A. (2019). Emotions at the border: Increased punishment behavior during fair interpersonal exchanges in borderline personality disorder. Journal of Abnormal Psychology, 128(2), 162172.

Decety, J. (2010) The neurodevelopment of empathy in humans. Dev Neurosci. 2010 Dec; 32(4): 257-267.

Decety, J., \& Jackson, P.L. (2006). A social-neuroscience perspective on empathy. Current Direction in Psychological Science, 15, 54-58

Dörfel, D., Lamke, J.P., Hummel, F., Wagner, U., Erk, S., Walter, H. (2014) Common and differential neural networks of emotion regulation by Detachment, Reinterpretation, Distraction, and Expressive Suppression: a comparative fMRI investigation. NeuroImage. 2014;101:298-309. doi: https://doi.org/10.1016/j.neuroimage.2014. 06.051 .

Drabant, E. M., McRae, K., Manuck, S. B., Hariri, A. R., and Gross, J. J. (2009). Individual differences in typical reappraisal use predict amygdala and prefrontal responses. Biol. Psychiatry 65, 367-373. doi: https://doi.org/10.1016/j.biopsych.2008.09.007

Etkin, A., Buchel, C., Gross, J.J. (2015) The neural bases of emotion regulation. Nature Reviews Neuroscience Vol 16, pp. 693-700 (2015)
Fonville, L., Giampietro, V., Surguladze, S., Williams, S., Tchanturia, K. (2013) Increased BOLD signal in the fusiform gyrus during implicit emotion processing in anorexia nervosa. Neuroimage Clin. 2013 Dec 7;4:266-73

Frick, A., Howner, K., Fischer, H., Kristiansson, M., Furmark, T. (2013) Altered fusiform connectivity during processing of fearful faces in social anxiety disorder. Transl Psychiatry. 2013 Oct; 3(10): e312.

Galili, T. (2015). Dendextend: an r package for visualizing, adjusting, and comparing trees of hierarchical clustering. Bioinformatics, 2015.

Gallagher, H.L., Happé, F., Brunswick, N., Fletcher, P.C., Frith, U., Frith C.D. Reading the mind in cartoons and stories: an fMRI study of 'theory of mind' in verbal and nonverbal tasks. Neuropsychologia. 2000; 38(1):11-21.

Giuliani, N.R., Drabant, E.M., Gross, J.J. (2011) Anterior cingulate cortex volume and emotion regulation: is bigger better? Biological Psychology, 86, 379-82

Goldin P.R., Manber-Ball T., Werner K., Heimberg R., Gross J.J. (2009) Neural mechanisms of cognitive reappraisal of negative self-beliefs in social anxiety disorder. Biol Psychiatry. 2009 Dec 15; 66(12): 1091-9.

Goldin, P.R., McRae, K., Ramel, W., Gross, J.J. The Neural Bases of Emotion Regulation: Reappraisal and Suppression of Negative Emotion. Biol Psychiatry 2008; 63:577-586

Grecucci, A., Sulpizio, S., Tommasello, E., Vespignani, F., Job, R. (2019) Seeing emotions, reading emotions: Behavioral and ERPs evidence of the regulation of pictures and words. PloS One 14(5): e0209461

Grecucci A., Rubicondo, D., Siugzdaite, R., Surian, L., Job, R. (2016) Uncovering social deficits in autistic individuals: A source-based morphometry study. Frontiers in Neuroscience, 31,10:388

Grecucci, A., Giorgetta, C., Van't Wout, M., Bonini, N., Sanfey, A.G. Reappraising the ultimatum: an fMRI study of emotion regulation and decision making (2013a) Cereb Cortex. 2013;23(2):399-410. doi: https://doi.org/10.1093/cercor/bhs028 .

Grecucci, A., Giorgetta, C., M. Bonini, Sanfey, NA (2013b). Reappraising social emotions: the role of inferior frontal gyrus, temporo-parietal junction and insula in interpersonal regulation. Frontiers in Human Neuroscience, 7, 523.

Gross, J. (1999). Emotion regulation: Past, present, future. Cogn Emot. 13(5):551-573

Gross, J. J. (1998). Antecedent- and response-focused emotion regulation: Divergent consequences for experience, expression, and physiology. Journal of Personality and Social Psychology, 74, 224-237

Gross, J. J., \& John, O. P. (2003) Individual Differences in Two Emotion Regulation Processes: Implications for Affect, Relationships, and Well-being. Journal of Personality and Social Psychology, 2003, Vol. 85, No. 2, 348-362

Gruber, J., Hay, A.C., Gross, J.J. (2014). Rethinking emotion: cognitive reappraisal is an effective positive and negative emotion-regulation strategy in bipolar disorder. Emotion 14, 388-396.

Gunaydin, G., Selcuk, E., Ong, A.D. (2016) Trait reappraisal predicts affective reactivity to daily positive and negative events. Front Psychol 7: 1000

Hall, P., \& Wilson, S. R. (1991) Two guidelines for bootstrap hypothesis testing. Biometrics, 757-762.

Haxby, J.V., Hoffman, E.A., Gobbini, M.I. (2000) The distributed human neural system for face perception. Trends Cogn Sci. 2000 Jun; 4(6): 223-233.

Haxby, J.V., Hoffman, E.A., Gobbini, M.I. (2002) Human neural systems for face recognition and social communication. Biol Psychiatry. 2002 Jan 1; 51(1):59-67.

Hayes, J. P., Morey, R. A., Petty, C. M., Seth, S., Smoski, M. J., McCarthy, G., et al. (2010). Staying cool when things get hot: emotion regulation modulates neural mechanisms of memory encoding. Front. Hum. Neurosci. 4:230. doi: https://doi.org/10.3389/fnhum. 2010.00230 
Hermann, A., Bieber, A., Keck, T., Vaitl, D., Stark, R. Brain structural basis of cognitive reappraisal and expressive suppression. Soc Cogn Affect Neurosci. 2014;9(9):1435-42

Kanske, P., Heissler, J., Schönfelder, S., Bongers, A., Wessa, M. (2011) How to Regulate Emotion? Neural Networks for Reappraisal and Distraction. Cerebral Cortex, Vol 21, 6 pp. 1379-1388,

Koenigsberg, H.W., Siever, L.J., Lee, H., Pizzarello, S., New, A.S., Goodman, M., Cheng, H., Flory, J., Prohovnik, I. (2009) Neural correlates of emotion processing in borderline personality disorder. Psychiatry Res. 2009 Jun 30; 172(3): 192-199.

Krause-Utz A., Winter, D., Niedtfeld, I., Schmahl, C. (2014) The latest neuroimaging findings in borderline personality disorder. Curr Psychiatry Rep. 2014 Mar;16(3):438

Kring A.M., \& Werner K.H. (2004). The Regulation of Emotion. Lawrence Erlbaum Associates Publishers; Mahwah, NJ, Mahwah, NJ, US. Emotion regulation and psychopathology. pp. 359-385.

Kühn, S., Vanderhasselt, M.A., De Raedt, R., Gallinat, J. (2014) The neural basis of unwanted thoughts during resting state. Soc Cogn Affect Neurosci. 2014 Sep; 9(9): 1320-1324

Laghi, F., Lonigro, A., Pallini, S., Baiocco, R. (2018) Emotion Regulation and Empathy: Which Relation with Social Conduct? J Genet Psychol. 2018 Jan 31:1-9. doi: https://doi.org/10.1080/00221325. 2018.1424705.

Lebowitz, M.S., \& Dovidio, J.F. (2015) Implications of emotion regulation strategies for empathic concern, social attitudes, and helping behavior. Emotion, 15(2), 187-194. doi: https://doi.org/10.1037/ a0038820

Lindquist, M.A. \& Gelman, A. (2009) Correlations and Multiple Comparisons in Functional Imaging: A Statistical Perspective (Commentary on Vul et al., 2009). Perspect Psychol Sci. 2009;4(3):310-3. doi: https://doi.org/10.1111/j.1745-6924.2009. 01130.x .

Lockwood, P.L., Seara-Cardoso, A., Viding, E. (2014). Emotion regulation moderates the association between empathy and prosocial behavior. PLoS One, 9, e96555

Martin, L.L., \& Tesser, A. (1996) Striving and feeling: Interactions among goals, affect, and self-regulation. Hillsdale, NJ: Lawrence Erlbaum

McRae, K., Gross, J.J., Weber, J., Robertson, E.R., Sokol-Hessner, P., Ray, R.D, Gabrieli, J.D.E., Ochsner, K.N. The development of emotion regulation: an fMRI study of cognitive reappraisal in children, adolescents and young adults. Soc Cogn Affect Neurosci. 2012; 7(1): $11-22$

McRae, K., Hughes, B., Chopra, S., Gabrieli, J.D.E. (2010) The Neural Bases of Distraction and Reappraisal. Journal of Cognitive Neuroscience 22:2, pp. 248-262

Nunes, P.M., Wenzel, A., Borges, K.T., Porto, C.R., Caminha, R.M., de Oliveira I.R. (2009) Volumes of the Hippocampus and Amygdala in Patients With Borderline Personality Disorder: A Meta-Analysis. Journal of Personality Disorders 23, (4), 333-345

Messina, I., Bianco, S., Sambin, M., Viviani, R. (2015). Executive and semantic processes in reappraisal of negative stimuli: insights from a meta-analysis of neuroimaging studies. Frontiers in Psychology, 6, 956

Messina, I., Sambin, M., Beschoner, P., Viviani, R. (2016). Changing views of emotion regulation and neurobiological models of the mechanism of action of psychotherapy. Cogn, Affect, \& Behav Neurosci, 16(4), 571-587.

Meyer, T., Smeets, T., Giesbrecht, T., Merckelbach, H. (2012) The efficiency of reappraisal and expressive suppression in regulating everyday affective experiences. Psychiatry Research 200 (2012) 964969

Miller, D.J., Vachon, D.D., Aalsma, N.C. (2012) Negative affect and emotion dysregulation. Conditional Relations With Violence and Risky Sexual Behavior in a Sample of Justice-Involved
Adolescents. Criminal Justice and Behavior, Vol. 39, No. 10, 1316-1327

Nolen-Hoeksema S., Wisco, B.E., Lyubomirsky, S. (2008) Rethinking rumination. Perspectives on Psychological Science. 2008;3:400424.

Ochsner, K. N., and Gross, J. J. (2005). The cognitive control of emotion. Trends Cogn. Sci. 9, 242-249. doi: https://doi.org/10.1016/j.tics. 2005.03.010

Ochsner, K. N., Silvers, J. A., Buhle, J. T. (2012). Functional imaging studies of emotion regulation: a synthetic review and evolving model of the cognitive control of emotion. Ann. N YAcad. Sci. 1251, E1E24. doi: https://doi.org/10.1111/j.1749-6632.2012.06751.x

O'Neil A., D'Souza A., Samson A.C., Carballedo, A., Kerskens, C., Frodl, T. (2015) Dysregulation between emotion and theory of mind networks in borderline personality disorder. Psychiatry Res. 2015;231(1):25-32.

Pappaianni, E., Siugzdaite, R., Vettori, S., Venuti, P., Job, R., Grecucci, A. (2018) Three shades of gray: detecting brain abnormalities in children with autism using source-, voxel- and surface-based morphometry. Eur J Neurosci, pp. 1-11, 47(6):690-700

Patterson, K., Peter, J., Nestor, P. J., Timothy, T., Rogers, T. T. (2007) Where do you know what you know? The representation of semantic knowledge in the human brain. Nat. Rev. Neurosci. 8, 976-987

Rive, M.M., van Rooijen, G., Veltman, D.J., Phillips, M.L., Schene, A.H., Ruhé, H.G. (2013) Neural correlates of dysfunctional emotion regulation in major depressive disorder. A systematic review of neuroimaging studies. Neurosci Biobehav Rev. 2013;37(10 Pt 2):2529-53

Samson, D., Apperly, I.A., Chiavarino, C., Humphreys, G.W. (2004) Left temporoparietal junction is necessary for representing someone else's belief. Nat Neurosci. 2004;7(5):499-500.

Saxe, R., Kanwisher, N. (2003) People thinking about thinking people. The role of the temporo-parietal junction in "theory of mind". NeuroImage. 2003 Aug; 19(4):1835-42.

Schipper, M. \& Petermann, F. (2013). Relating empathy and emotion regulation: Do deficits in empathy trigger emotion dysregulation? Soc Neurosci. 2013;8(1):101-7.

Schulze, L., Schmahl, C., Niedtfeld, I. Neural Correlates of Disturbed Emotion Processing in Borderline Personality Disorder: A Multimodal Meta-Analysis. Biol Psychiatry. 2016;79(2):97-106

Segall, J. M., Allen, E. A., Jung, R. E., Erhardt, E. B., Arja, S. K., Kiehl, K., Calhoun, V.D. (2012) Correspondence between structure and function in the human brain at rest. Frontiers in Neuroinformatics. 2012 Mar 27;6:10

Senkowski, D., Kautz, J., Hauck, M., Zimmermann, R., Engel, A.K. (2011) Emotional facial expressions modulate pain-induced beta and gamma oscillations in sensorimotor cortex. $J$ Neurosci. 2011;31(41):14542-50

Shamay-Tsoory, S.G. The neural bases of empathy. Neuroscientist 2011;17(1):18-24.

Sheppes, G., Suri, G., Gross, J.J. (2015) Emotion regulation and psychopathology. Annu Rev Clin Psychol. 2015;11:379-405

Srivastava, S., Tamir, M., McGonigal, K.M., John, O.P., Gross, J.J. (2009). The social costs of expressive suppression: A prospective study of the transition to college. Journal of Personality and Social Psychology, 96, 883-897.

Terracciano, A., McCrae, R.R., Costa, P.T.J. Factorial and construct validity of the Italian Positive and Negative Affect Schedule (PANAS). Eur J Psychol Assess. 2003; 19(2): 131-141

Uchida, M., Biederman, J., Gabrieli, J.D.E., Micco, J., de Los Angeles, C., Brown, A., Kenworthy, T., Kagan, E., Whitfield-Gabrieli, S. (2015) Emotion regulation ability varies in relation to intrinsic functional brain architecture. Soc Cogn Affect Neurosci. 2015 Dec; 10(12): 1738-1748.

Vanderhasselt, M. A., Baeken, C., Van Schuerbeek, P., Luypaert, R., and De Raedt, R. (2013). Inter-individual differences in the habitual use of cognitive reappraisal and expressive suppression are associated 
with variations in prefrontal cognitive control for emotional information: an event related fMRI study. Biol. Psychol. 92, 433-439. doi: https://doi.org/10.1016/j.biopsycho.2012.03.005.

Victor, Y. J., Ricardo, M. A. (1990) The maximum bias of robust covariances," Communications in Statistics-Theory and Methods, vol. 19, no. 10 , pp. 3925-3933.

Visintin, E., De Panfilis, C., Amore, M., Balestrieri, M., Wolf, R.C., Sambataro, F. (2016) Mapping the brain correlates of borderline personality disorder: A functional neuroimaging meta-analysis of resting state studies. $J$ Affect Disord. 2016 Nov 1;204:262-9

Viviani, R. (2013). Emotion regulation, attention to emotion, and the ventral attentional network. Frontiers in human neuroscience, 7, 746.

Vul, E., Harris, C., Winkielman, P., Pashler, H. Puzzlingly High Correlations in fMRI Studies of Emotion, Personality, and Social Cognition. Perspect Psychol Sci. 2009;4(3):274-90. doi: https://doi. org/10.1111/j.1745-6924.2009.01125.x

Watson, D., Clark, L.A., Tellegen, A. (1988). Development and validation of brief measures of positive and negative affect: The PANAS scales. Journal of Personality and Social Psychology, 54, 10631070.

Webb, T.L., Miles, E., Sheeran, P. (2012). Dealing with feeling: a metaanalysis of the effectiveness of strategies derived from the process model of emotion regulation. Psychol. Bull. 138, 775-808
Welborn, B.L., Papademetris, X., Reis, D.L., Rajeevan, N., Bloise, S.M., Gray, J.R. (2009) Variation in orbitofrontal cortex volume: relation to sex, emotion regulation and affect. Soc Cogn Affect Neurosci. 2009;4(4):328-39. doi: https://doi.org/10.1093/scan/nsp028

Winecoff, A., LaBar, K.S., Madden, D.J., Cabeza, R., Huettel, S.A. (2011). Cognitive and neural contributors to emotion regulation in aging. Soc. Cogn. Affect. Neurosci. 6, 165-176. doi: https://doi.org/ $10.1093 / \mathrm{scan} / \mathrm{nsq} 030$

Xu, L., Groth, K.M., Pearlson, G., Schretlen, D.J., Calhoun, V.D. (2009) Source-based morphometry: the use of independent component analysis to identify gray matter differences with application to schizophrenia. Hum. Brain Mapp., 30, 711-724.

Yarkoni, T. Big Correlations in Little Studies: Inflated fMRI Correlations Reflect Low Statistical Power Commentary on Vul et al. (2009). Perspect Psychol Sci. 2009;4(3):294-8. doi: https://doi.org/10. 1111/j.1745-6924.2009.01127.x.

Publisher's note Springer Nature remains neutral with regard to jurisdictional claims in published maps and institutional affiliations. 